\title{
Buscando el punto crítico de presión arterial sistólica para la oclusión endovascular de la aorta: Análisis mundial de los registros REBOA
}

\author{
Finding the optimal critical threshold value of systolic blood pressure for \\ endovascular aortic occlusion: A worldwide analysis of REBOA databases
}

Helmer Palacios ${ }^{1} \mathbb{D}$, Christian Delgado ${ }^{1}$, Carlos Munar ${ }^{1}$, Yaset Caicedo ${ }^{2} \mathbb{D}$, Alexander Salcedo ${ }^{3,4} \mathbb{D}$, José Julián Serna ${ }^{3,4} \mathbb{D}$, Fernando Rodríguez-Holguín ${ }^{3} \mathbb{D}$,

Alberto García ${ }^{3,4} \mathbb{D}$, Carlos Serna ${ }^{1}$, Michael Parra ${ }^{5} \mathbb{D}$, Carlos Ordoñez $^{3,4} \mathbb{D}$

1 MD, especialista en Cirugía general, fellow Cirugía de Trauma y Emergencias, Universidad del Valle, Cali, Colombia.

2 Centro de Investigaciones Clínicas, Fundación Valle del Lili, Cali, Colombia

3 MD, especialista en Cirugía general, División de Cirugía de Trauma y Emergencias, departamento de Cirugía General, Fundación Valle del Lili, Cali, Colombia.

4 Sección de Cirugía de Trauma y Emergencias, departamento de Cirugía General, Hospital Universitario del Valle Evaristo García, Universidad del Valle, Cali, Colombia.

5 MD, FACS, Department of Trauma and Acute Care Surgery, Broward General Level I Trauma Center, Fort Lauderdale, USA.

Trabajo ganador del segundo puesto en el Concurso de Investigación en Cirugía "José Félix Patiño Restrepo" del Congreso Semana Quirúrgica Nacional 2020 de la Asociación Colombiana de Cirugía. Bogotá, D.C, Colombia. Noviembre de 2020.

\section{Resumen}

Introducción. La presión arterial sistólica puede ser un factor determinante para la toma de decisiones en el manejo de pacientes con trauma severo y hemorragia no compresible del torso. El objetivo de este trabajo fue determinar el punto óptimo de presión arterial sistólica previo a la oclusión endovascular de aorta asociado con la mortalidad a las 24 horas.

Métodos. Se realizó un análisis combinado de dos bases de datos de registro de REBOA, ABO-Trauma Registry y AAST-AORTA, que incluye pacientes de Norte América, Suramérica, Europa, Asia y África. Pacientes sin efecto hemodinámico con el uso del REBOA fueron excluidos. Se describieron las características demográficas, clínicas y de la colocación del REBOA en los pacientes que fallecieron en las primeras 24 horas. Se analizó la asociación entre la presión arterial sistólica previa a la oclusión aortica y la mortalidad a través de modelos de regresión logística y se evaluó el poder predictivo de la presión arterial sistólica en un intervalo entre 60 y $90 \mathrm{mmHg}$.

Resultados. Fueron identificados 871 registros, pero solo 693 pacientes cumplieron con los criterios de inclusión. El trauma cerrado se presentó en el $67,2 \%$ de los pacientes y la severidad del trauma tuvo una mediana de ISS

Fecha de recibido: 15/09/2020 - Fecha de aceptación: 15/11/2020 - Fecha de publicación en línea: 10/02/2021

Correspondencia: Carlos A. Ordoñez, Carrera 98 \#18-49, Fundación Valle del Lili, Cali, Colombia

Dirección electrónica: ordonezcarlosa@gmail.com / carlos.ordonez@fvl.org.co. Telefono: 3006319118

Citar como: Palacios H, Delgado C, Munar C, Caicedo Y, Salcedo A, Serna JJ, et al. Buscando el punto crítico de presión arterial sistólica para la oclusión endovascular de la aorta: Análisis mundial de los registros REBOA. Rev Colomb Cir. 2021;36:237-47. https://doi.org/10.30944/20117582.840

Este es un artículo de acceso abierto bajo una Licencia Creative Commons - BY-NC-ND https://creativecommons.org/licenses/by-ncnd/4.0/deed.es 
de 34 (RIQ: 25-45). La mediana de la presión arterial sistólica previa al REBOA fue de 61 mmHg (RIQ: 46-80). La mortalidad a las 24 horas fue del 34,6 \%. La asociación entre la presión arterial sistólica pre-oclusión de la aorta y la mortalidad a las 24 horas tiene una capacidad predictiva de acuerdo con el área bajo la curva ROC para trauma cerrado de 0,64 (IC $\left.{ }_{95 \%} 0,59-0,70\right)$ y para trauma penetrante de $0,61\left(\mathrm{IC}_{95 \%} 0,53-0,69\right)$. Se identificó que la presión arterial sistólica de $70 \mathrm{mmHg}$ se asocia con un aumento por encima del $25 \%$ de la mortalidad a las 24 horas.

Discusión. La presión arterial sistólica de $70 \mathrm{mmHg}$ en pacientes con trauma severo y hemorragia no compresible puede ser el punto crítico para la oclusión endovascular de aorta para mejorar la supervivencia de los pacientes, sin importar el mecanismo de trauma. Sin embargo, la presión arterial sistólica debe complementarse con otros factores clínicos para tomar la decisión oportuna.

Palabras clave: aorta; procedimientos endovasculares; oclusión con balón; heridas y lesiones; hemorragia; presión sanguínea; mortalidad; REBOA.

\begin{abstract}
Introduction. Systolic blood pressure (SBP) can be a determining factor for decision-making in the management of patients with severe trauma and non-compressible torso bleeding (NTCH). The objective of this study was to determine the optimal SBP threshold value prior to endovascular occlusion of the aorta associated with 24-hour mortality.
\end{abstract}

Methods. A combined analysis of two REBOA registry databases, ABO-Trauma Registry and AAST-AORTA, was performed, which includes patients from North America, South America, Europe, Asia, and Africa. Patients without hemodynamic effect with the use of REBOA were excluded. Demographic, clinical, and REBOA placement characteristics were described in patients who died in the first 24 hours. The association between SBP prior to aortic occlusion and mortality was analyzed using logistic regression models and the predictive power of SBP was evaluated in an interval between 60 and $90 \mathrm{mmHg}$.

Results. 871 records were identified, but only 693 patients met the inclusion criteria. Blunt trauma occurred in $67.2 \%$ of the patients and the severity of the trauma had a median ISS of 34 (IQR: 25-45). The median systolic blood pressure prior to REBOA was $61 \mathrm{mmHg}$ (IQR: 46-80). The 24-hour mortality was 34.6\%. The association between systolic blood pressure pre-occlusion of the aorta and mortality at 24 hours has a predictive capacity according to the area under the ROC curve for blunt trauma of 0.64 (95\% CI: 0.59-0.70) and for penetrating trauma of 0.61 (95\% CI: 0.53-0.69). A systolic blood pressure of $70 \mathrm{mmHg}$ was found to be associated with an increase of over $25 \%$ in mortality at 24 hours.

Discussion. SBP of $70 \mathrm{mmHg}$ in patients with severe trauma and non-compressible hemorrhage may be the critical point for endovascular aortic occlusion to improve patient survival, regardless of the mechanism of trauma. However, systolic blood pressure must be supplemented with other clinical factors to make the timely decision.

Keywords: aorta; endovascular procedures; balloon occlusion; wounds and injuries; hemorrhage; blood pressure; mortality; REBOA.

\section{Introducción}

La hemorragia masiva es la principal causa de muerte prevenible en pacientes con trauma y hemorragia no compresible del torso, ocasionando del 60 al $70 \%$ de los fallecimientos ${ }^{1,2}$. El rápido control de la hemorragia es esencial en estas situaciones y la demora en las intervenciones se asocia con un aumento en la mortalidad ${ }^{3,4}$.
La oclusión de la aorta es una medida temporal para controlar la tasa de exanguinación y aumentar la perfusión cerebral y coronaria ${ }^{5}$. Clásicamente, se ha empleado este procedimiento en el paciente moribundo por vía torácica o abdominal, pero recientemente, se ha logrado a través del balón de reanimación endovascular de aorta (en inglés, $R e$ suscitative Endovascular Balloon Occlusion of the 
Aorta, REBOA), permitiendo un acceso menos invasivo, la posibilidad de intervenciones más tempranas y la disponibilidad de una herramienta activa y dinámica en los esfuerzos de resucitación ${ }^{6,7}$.

Actualmente es un desafío definir en qué momento se debe intervenir con el uso del REBOA en los pacientes con trauma y choque hemorrágico severo para aumentar su supervivencia. Las indicaciones de la oclusión de la aorta son paro cardiaco prehospitalario, con maniobras de reanimación cardiopulmonar que no excedan los 15 minutos en trauma penetrante torácico y 10 minutos para trauma cerrado. Sin embargo, estas indicaciones aplican para los pacientes con colapso hemodinámico, en quienes la oclusión de la aorta se considera como última medida de rescate ${ }^{8}$.

El REBOA permite realizar una intervención más temprana y hacer un control transitorio de la lesión para evitar el paro cardiaco ${ }^{9,10}$. Para identificar la posible inestabilidad hemodinámica en pacientes con hemorragia masiva se ha postulado como parámetro clínico a la presión arterial sistólica (PAS). El American College of Surgeons ha propuesto tener como referente en la evaluación inicial una PAS $<90 \mathrm{mmHg}$ para la toma de decisiones ${ }^{11}$. No obstante, para decidir si se realiza la oclusión de la aorta u otras medidas agresivas en la reanimación, este valor pueden ser controversiales $9,12,13$. Se ha postulado que identificar un valor de PAS para la oclusión de la aorta se podría asociar con un aumento en la supervivencia.

Con su experiencia local en el uso del REBOA en un único centro de alta complejidad, previamente los autores han explorado esta hipótesis, encontrando que una PAS menor a $60 \mathrm{mmHg}$ al momento del ingreso se asocia a una mortalidad a las 24 horas superior del $50 \%{ }^{14,15}$. El objetivo de este estudio fue determinar el punto óptimo de PAS previo a la oclusión de la aorta mediante REBOA en los pacientes con trauma severo y hemorragia no compresible del torso.

\section{Métodos}

\section{Diseño del estudio}

Se hizo un análisis combinado de dos bases de datos de recolección prospectiva respecto al manejo del REBOA.
El ABO-Trauma registry es una base de datos internacional, multicéntrica, observacional, prospectiva, financiada por el Departamento de Cirugía Cardiotorácica y Vascular del Hospital Universitario de Örebro (Örebro, Suecia) y el grupo de investigación Endovascular and Trauma Management (EVTM). Este registro incluye centros hospitalarios de Rusia, Israel, Suecia, Finlandia, Japón, Italia, Corea del Sur, Tailandia, Suráfrica, Alemania, Colombia, Turquía y Holanda. Se incluyeron los registros recolectados entre julio de 2014 y diciembre de 2019.

El registro Aortic Occlusion for Resuscitation in Trauma and Acute Care Surgery (AORTA), desarrollado por la American Association for the Surgery of Trauma (AAST), es un registro multicéntrico, observacional, prospectivo, que evalúa el uso de la oclusión de aorta en la resucitación de pacientes con trauma severo hemodinámicamente inestables. Los sujetos de la base de datos del AAST-AORTA fueron recolectados entre noviembre de 2013 y enero de 2020, y se incluyeron únicamente los pacientes que fueron sometidos a oclusión de aorta a través de la colocación del REBOA.

Las variables similares de las dos bases de datos fueron combinadas en una única base para su análisis, de manera similar al trabajo previo de Duchesne y colaboradores ${ }^{10}$. Adicionalmente, se incluyó la información de los requerimientos de resucitación, como volumen de cristaloides y hemoderivados transfundidos en las primeras 24 horas, de la base de datos del AAST-AORTA. Se excluyeron aquellos pacientes con PAS de $0 \mathrm{mmHg}$ previa a la oclusión de aorta y que después de la oclusión se mantuviera así.

\section{Variables}

La información demográfica y clínica recolectada fue: edad, género, mecanismo de trauma, severidad del trauma por Injury Severity Score (ISS), localización anatómica de la lesión, paro cardiaco prehospitalario, signos vitales y paraclínicos al ingreso, detalles técnicos en la colocación del REBOA, zona de la oclusión de la aorta, tiempo de la oclusión de aorta, PAS previa y posterior a la inserción del REBOA, mortalidad a las 24 horas y mortalidad intrahospitalaria. 
El politrauma fue definido como dos o más regiones anatómicas lesionadas. El delta de la PAS fue definido como la diferencia entre la PAS posterior a la inserción y la PAS previa a la inserción. La no respuesta hemodinámica se consideró como aquel paciente con PAS previa a la inserción mayor a la PAS posterior a la inserción del REBOA.

\section{Análisis estadístico}

Las variables categóricas fueron reportadas como frecuencia absoluta y frecuencia relativa. Las variables continuas fueron descritas usando mediana y rango intercuartil (RIQ). Para las variables categóricas, sus diferencias fueron evaluadas mediante la prueba de Chi-cuadrado o prueba exacta de Fisher. Las variables continuas fueron comparadas mediante la prueba de U-Mann-Whitney. Se compararon los pacientes con mortalidad a las 24 horas y los sobrevivientes.

Para determinar la relación entre la PAS previa a la oclusión de la aorta y la mortalidad a las 24 horas se exploraron modelos de regresión logística univariado, según el mecanismo de trauma y una relación de los datos de tipo lineal, cuadrática o cubica. La evaluación del modelo fue analizada mediante la prueba de Chi-cuadrado de Hosmer-Lemeshow y se usó el Bayesian Criterion Information (BIC) para escoger el mejor modelo según principio de parsimonia. Se definió como punto de corte clínicamente relevante aquel punto de PAS asociado a un valor de mortalidad estimada a las 24 horas superior al $25 \%$.

Para evaluar la capacidad discriminatoria del umbral de PAS en predecir la mortalidad a 24 horas, se realizó un análisis de curvas ROC (Receiver Operating Characteristics) y se calculó el área bajo la curva (Area Under Curve - AUC). El mejor punto de corte de PAS, con respecto a la sensibilidad y especificidad, se estimó por medio del índice de Youden ${ }^{16}$. Sin embargo, se encontraron estimaciones por debajo de $60 \mathrm{mmHg}$, lo cual podrían tener poca aplicabilidad en el contexto clínico, por lo que se evaluaron los puntos de corte de PAS en el rango de 60 a $90 \mathrm{mmHg}$, por cada $10 \mathrm{mmHg}$, mediante sensibilidad, especificidad, valores predictivos, likelihood ratio y odds ratio. Se calcularon intervalos de confianza al $95 \%$ ( $\left.\mathrm{IC}_{95 \%}\right)$. El análisis estadístico se realizó usando R-Language versión 3.6.3 ${ }^{17}$.

\section{Resultados \\ Características generales}

Un total de 871 registros fueron obtenidos de las bases de datos, 184 registros del ABO-Trauma registry y 687 registros del AAST-AORTA. Se excluyeron 178 pacientes por presentar información incompleta de PAS previa o posterior a la colocación del REBOA, o porque la PAS se mantuvo en $0 \mathrm{mmHg}$ a pesar de la colocación del REBOA. Se incluyeron en el análisis 693 pacientes, cuyas características generales fueron: 517 (74,6\%) eran hombres, con una mediana de edad de 42 años (RIQ: 27-58), el mecanismo de trauma más común fue el cerrado reportado en 466 (67,2\%) pacientes, y la mediana de ISS fue de 34 (RIQ: 25-45).

La mortalidad a las 24 horas se presentó en 240 (34,6 \%) pacientes. Las características de los pacientes fallecidos en las primeras 24 horas versus los supervivientes están resumidas en las tablas 1 y 2 . La proporción de pacientes con paro cardiaco prehospitalario fue mayor en el grupo de mortalidad a las 24 horas $(91,37,9 \%)$ que entre los supervivientes $(57,12,6 \%)$, con una diferencia estadísticamente significativa $(\mathrm{p}<0.001)$. Como se aprecia en la tabla 1, los parámetros clínicos y paraclínicos de los pacientes que fallecieron en las primeras 24 horas fueron más severamente alterados. La mortalidad intrahospitalaria en el grupo de los pacientes supervivientes a las 24 horas se reportó en 136 (30\%) pacientes.

Respecto a los criterios técnicos de la colocación del REBOA, entre los pacientes con acceso por la arteria femoral, el procedimiento se realizó en urgencias en $474(68,3 \%)$ pacientes y la técnica más común fue la percutánea, en 326 (47\%) pacientes. La zona más común de oclusión de la aorta fue la zona 1 (471, 67,9\%). La mediana de PAS previa a la inserción del REBOA fue más alta en el grupo de los supervivientes (67, RIQ: 50-81 mmHg) que en el grupo de mortalidad a las 24 horas (53, RIQ: 0-72 $\mathrm{mmHg}$ ), con una diferencia estadísticamente significativa $(p<0.001)$. Adicio- 
Tabla 1. Características generales de la población de estudio

\begin{tabular}{|c|c|c|c|c|}
\hline & $\begin{array}{c}\text { Total } \\
(n=693)\end{array}$ & $\begin{array}{l}\text { Superviviente } \\
(n=453)\end{array}$ & $\begin{array}{l}\text { Mortalidad a las } 24 \text { horas } \\
\qquad(n=240)\end{array}$ & $\mathbf{p}$ \\
\hline Edad, mediana (RIQ) & $42(27-58)$ & $42(27,58)$ & $42(27-59)$ & 0,7 \\
\hline Masculino, n (\%) & $517(74,6)$ & $340(75,1)$ & $177(73,8)$ & 0,776 \\
\hline \multicolumn{5}{|l|}{ Mecanismo de trauma, $\mathrm{n}(\%)$} \\
\hline Cerrado & $466(67,2)$ & $298(65,8)$ & $168(70)$ & \multirow{3}{*}{0,214} \\
\hline Penetrante & $223(32,1)$ & $151(33,3)$ & $72(30)$ & \\
\hline Combinado & $4(0,5)$ & $4(0,9)$ & 0 & \\
\hline Injury Severity Score, mediana (RIQ) & $34(25-45)$ & $34(25-43)$ & $35(25-50)$ & 0,051 \\
\hline \multicolumn{5}{|l|}{ Localización anatómica del trauma, n (\%) } \\
\hline Craneoencefálico & $280(40,4)$ & $173(38,2)$ & $107(44,6)$ & 0,121 \\
\hline Tórax & $312(45,0)$ & $191(42,2)$ & $121(50,4)$ & 0,045 \\
\hline Abdomen & $439(63,3)$ & $295(65,1)$ & $144(60)$ & 0,211 \\
\hline Pelvis & $162(23,3)$ & $119(26,3)$ & $43(17,9)$ & 0,017 \\
\hline Politrauma & $392(56,5)$ & $255(56,3)$ & $137(57,1)$ & 0,904 \\
\hline Paro cardiaco prehospitalario, n (\%) & $148(21,3)$ & $57(12,6)$ & $91(37,9)$ & $<0,001$ \\
\hline Ausencia de respuesta pupilar, $\mathrm{n}(\%)$ & $296(42,7)$ & $143(31,6)$ & $153(63,7)$ & $<0,001$ \\
\hline PAS al ingreso, $\mathrm{mmHg}$, mediana (RIQ) & $70(10-102)$ & $81(70-110)$ & $70(0-95)$ & $<0,001$ \\
\hline \multicolumn{5}{|c|}{ Frecuencia cardiaca al ingreso, latidos por minuto, $\mathrm{n}(\%)$} \\
\hline$<50$ & $67(9,6)$ & $17(3,8)$ & $50(20,8)$ & \multirow{4}{*}{$<0,001$} \\
\hline $50-100$ & $142(20,4)$ & $96(21,2)$ & $46(19,2)$ & \\
\hline $101-120$ & $146(21,1)$ & $100(22,1)$ & $46(19,2)$ & \\
\hline$>120$ & $295(42,5)$ & $216(47,7)$ & $79(32,9)$ & \\
\hline $\mathrm{pH}$ al ingreso, mediana (RIQ) & $7,18(7,03-7,27)$ & $7,22(7,12-7,29)$ & $7,04(6,93-7,16)$ & $<0,001$ \\
\hline Lactato al ingreso, mg/dl, mediana (RIQ) & $6,7(4,1-10,9)$ & $5,6(3,7-8,7)$ & $10,4(6,7-13,6)$ & $<0,001$ \\
\hline Hemoglobina al ingreso, mg/dl, mediana (RIQ) & $10,9(8,4-12,4)$ & $11,4(9,7-12,8)$ & $10,4(8,4-12)$ & $<0,001$ \\
\hline INR al ingreso, mediana (RIQ) & $1,4(1,2-1,7)$ & $1,3(1,17-1,51)$ & $1,7(1,4-2,4)$ & $<0,001$ \\
\hline \multicolumn{5}{|l|}{ Mortalidad, n (\%) } \\
\hline Mortalidad a las 24 horas & $240(34,6)$ & - & - & \multirow{2}{*}{ NA } \\
\hline Intrahospitalaria & $375(54,1)$ & $136(30,0)$ & - & \\
\hline \multicolumn{5}{|l|}{ Lugar del fallecimiento, $\mathrm{n}(\%)$} \\
\hline Sala de Urgencias & $90(24)$ & $11(8,1)$ & $79(33,1)$ & \multirow{4}{*}{$<0,001$} \\
\hline Sala de Cirugía & $74(19,7)$ & $5(3,6)$ & $69(28,5)$ & \\
\hline Unidad de Cuidado Intensivo & $207(55,2)$ & $119(87,5)$ & $89(37,1)$ & \\
\hline Radiología Intervencionista & $2(0,5)$ & 0 & $2(0,8)$ & \\
\hline
\end{tabular}

${ }^{*}$ RIQ: Rango intercuartílico; PAS: Presión arterial sistólica; INR: índice internacional normalizado.

nalmente, 70 (29,2\%) de los pacientes fallecidos en 24 horas no tuvieron respuesta hemodinámica positiva con la colocación del REBOA. La mediana de la duración de la oclusión de aorta fue de 41 minutos (RIQ: 24-65).

\section{Asociación entre la PAS previa al REBOA y la mortalidad a las 24 horas}

Con la distribución de los datos, según los modelos de regresión logística de la PAS previa a la inser- ción del REBOA y la mortalidad a las 24 horas, se identificó que el mejor modelo para los pacientes con trauma cerrado tiene una relación cuadrática, mientras que para los pacientes con trauma penetrante tienen una relación lineal (tabla 3).

Respecto al trauma cerrado, la distribución de la mortalidad tiene un ascenso aproximado para valores de PAS previa al REBOA menores a 70 $\mathrm{mmHg}$ y por encima de $100 \mathrm{mmHg}$. La PAS previa a la inserción del REBOA en que la mortalidad 
Tabla 2. Características relacionadas con la colocación del REBOA y los requerimientos de reanimación

\begin{tabular}{|c|c|c|c|c|}
\hline & Total $(n=693)$ & $\begin{array}{l}\text { Superviviente } \\
\qquad(n=453)\end{array}$ & $\begin{array}{c}\text { Mortalidad a } \\
\text { las } 24 \text { horas } \\
(n=240)\end{array}$ & $\mathbf{p}$ \\
\hline \multicolumn{5}{|l|}{ Lugar del acceso femoral, n (\%) } \\
\hline Sala de Urgencias & $474(68,3)$ & $290(64)$ & $184(76,7)$ & \multirow{4}{*}{0,005} \\
\hline Sala de Cirugía & $183(26,4)$ & $136(30)$ & $47(19,6)$ & \\
\hline Radiología Intervencionista & $17(2,4)$ & $12(2,6)$ & $5(2,1)$ & \\
\hline Sin Dato & $19(2,7)$ & $15(3,3)$ & $4(1,7)$ & \\
\hline \multicolumn{5}{|l|}{ Método de Acceso Arterial, n (\%) } \\
\hline Percutáneo & $326(47,0)$ & $224(49,4)$ & $102(42,5)$ & \multirow{5}{*}{$<0,001$} \\
\hline Guiado por ecografía & $208(30,0)$ & $149(32,6)$ & $59(24,6)$ & \\
\hline Fluoroscopia & $11(1,5)$ & $9(2,0)$ & $2(0,8)$ & \\
\hline Técnica abierta & $128(18,4)$ & $55(12,6)$ & $73(30,4)$ & \\
\hline Sin Dato & $20(2,8)$ & $16(3,5)$ & $4(1,7)$ & \\
\hline \multicolumn{5}{|l|}{ Zona de oclusión de la Aorta, n (\%) } \\
\hline 1 & $471(67,9)$ & $277(61,1)$ & $194(80,8)$ & \multirow{3}{*}{$<0,001$} \\
\hline 2 & $14(2,0)$ & $11(2,4)$ & $3(1,2)$ & \\
\hline 3 & $208(30,0)$ & $165(36,4)$ & $43(17,9)$ & \\
\hline \multicolumn{5}{|l|}{ Personal encargado del REBOA, $n(\%)$} \\
\hline Cirujano de Trauma & $482(69,5)$ & $316(69,8)$ & $166(69,2)$ & \multirow{6}{*}{0,1} \\
\hline Fellow de Trauma & $55(7,9)$ & $34(7,5)$ & $21(8,8)$ & \\
\hline Residente de Cirugía general & $9(1,2)$ & $5(1,1)$ & $4(1,7)$ & \\
\hline Cirujano Vascular & $32(4,6)$ & $26(5,7)$ & $6(2,5)$ & \\
\hline Radiólogo & $23(3,3)$ & $15(3,3)$ & $8(3,3)$ & \\
\hline Emergenciólogo & $59(8,5)$ & $9(2,0)$ & $2(0,8)$ & \\
\hline PAS previa a inserción, mmHg, mediana (RIQ) & $61(46-80)$ & $67(50-81)$ & $53(0-72)$ & $<0,001$ \\
\hline PAS posterior a inserción, $\mathrm{mmHg}$, mediana (RIQ) & $104(87-125)$ & $108(92-125)$ & $95(50-122)$ & $<0,001$ \\
\hline No respuesta hemodinámica, n (\%) & $111(16,0)$ & $41(9,1)$ & $70(29,2)$ & $<0,001$ \\
\hline Delta de PAS, mediana (RIQ) & $36(15-61)$ & $36(20-60)$ & $36(0-66)$ & 0,064 \\
\hline Tiempo de oclusión de la Aorta, minutos, mediana (RIQ) & $41(24-65)$ & $40(25-64)$ & $45(23-67)$ & 0,407 \\
\hline \multicolumn{5}{|l|}{ Requerimientos de resucitación } \\
\hline Total cristaloides, Litros, mediana (RIQ) & $3(2-6)$ & $4(2-6)$ & $2(1-4)$ & $<0,001$ \\
\hline Unidades de glóbulos rojos empaquetados, mediana (RIQ) & $13(7-23)$ & $12(7-22)$ & $14(5-24)$ & 0,818 \\
\hline Unidades de plaquetas, mediana (RIQ) & $3(1-7,5)$ & $2(1-6)$ & $2(0-5)$ & 0,151 \\
\hline Unidades de plasma fresco congelado, mediana (RIQ) & $9(4-13)$ & $7(4-13)$ & $9(3-16)$ & 0,396 \\
\hline
\end{tabular}

${ }^{*} \mathrm{RIQ}$ : Rango intercuartílico; PAS: Presión arterial sistólica.

Tabla 3. Evaluación de los modelos de regresión logística de la presión arterial sistólica previa a la inserción del REBOA y la mortalidad a las 24 horas en los pacientes con trauma

\begin{tabular}{lccc}
\hline Mecanismo de trauma & $\begin{array}{c}\text { Tipo de modelo de regresión } \\
\text { logística }\end{array}$ & $\begin{array}{c}\text { Prueba de bonda de ajuste } \\
\text { (Chi cuadrado - Valor } \mathbf{p})\end{array}$ & BIC \\
\hline \multirow{3}{*}{ Trauma cerrado } & Lineal & $23,7-0,003$ & 593,1 \\
& Cuadrática & $7,02-0,531$ & 577,9 \\
Trauma penetrante & Cubica & $6,62-0,577$ & 582,9 \\
& Lineal & $2,50-0,961$ & 282,7 \\
& Cuadrática & $7,56-0,476$ & 284,6 \\
\hline
\end{tabular}

*BIC: Bayesian Criterion Information 
era superior al $25 \%$ en los pacientes con trauma cerrado fue de $84 \mathrm{mmHg}$ (Probabilidad estimada $25,1 \%, \mathrm{IC}_{95 \%}$ 20,2-30,3) y en los pacientes con trauma penetrante fue de $85 \mathrm{mmHg}$ (Probabilidad estimada $25,4 \%$, IC ${ }_{95 \%}$ 18-32,8).

En cuanto a los valores de PAS prácticos, se estimó la probabilidad asociada en el modelo para los puntos de corte de 60 y $70 \mathrm{mmHg}$. La PAS de 60 mmHg previo a la colocación del REBOA se asoció con una mortalidad del 28,1 \% ( IC $_{95 \%} 23,1-33,2$ ) en trauma cerrado y de $32 \%\left(\mathrm{IC}_{95 \%} 25,6-38,4\right)$ en trauma penetrante. En cambio, una PAS de 70 mmHg se asoció con una mortalidad del 26,1\% (IC ${ }_{95 \%}$ 21,3-31,0) para trauma cerrado y $29,2 \%$ (IC ${ }_{95 \%} 22,7-35,8$ ) en trauma penetrante (Figura 1).

\section{Rendimiento pronóstico de los puntos de corte de la PAS previa al REBOA}

Las curvas ROC según los mecanismos de trauma se muestran en la figura 2 . El área bajo la curva de la PAS para la mortalidad en 24 horas en trauma cerrado fue de 0,646 (IC I5\% $\left._{0} 0,591-0,701\right)$ y en trauma penetrante fue de 0,618 ( $\mathrm{IC}_{95 \%} 0,537$ $0,699)$. El punto de corte de acuerdo con el índice de Youden fue para trauma cerrado de $41 \mathrm{mmHg}$ y para trauma penetrante de $47 \mathrm{mmHg}$. No obstante, con el objetivo de describir un punto de corte con aplicabilidad clínica, se evaluó en un rango de PAS entre 60 y $90 \mathrm{mmHg}$ por cada $10 \mathrm{mmHg}$. La tabla 4 resume la sensibilidad, especificidad, valores predictivos, likelihood ratios y odds ratios con cada punto de corte.

La PAS previa al REBOA de $70 \mathrm{mmHg}$ se relaciona con un aumento de la mortalidad mayor del $25 \%$, tanto en trauma cerrado como en trauma penetrante. Este valor, en el trauma cerrado tuvo una especificidad del $42 \%$ ( $\mathrm{IC}_{95 \%} 36-47 \%$ ), un valor predictivo positivo de $42 \%$ (IC $_{95 \%} 36-47 \%$ ), con un OR de 2,00 (IC I5\% $_{9}$ 1,30-3,11). En cambio, para trauma penetrante tuvo una especificidad del $38 \%$ ( $\mathrm{IC}_{95 \%} 31-47$ ), con un valor predictivo positivo de $36 \%$ ( IC $_{95 \%}$ 29-45) y un OR de 1,73 $\left(\mathrm{IC}_{95 \%} 0,90-3,42\right)$.

\section{Discusión}

El manejo de pacientes con hemorragia no compresible del torso exsanguinante es un desafío ya que definir quién puede beneficiarse de la oclusión de
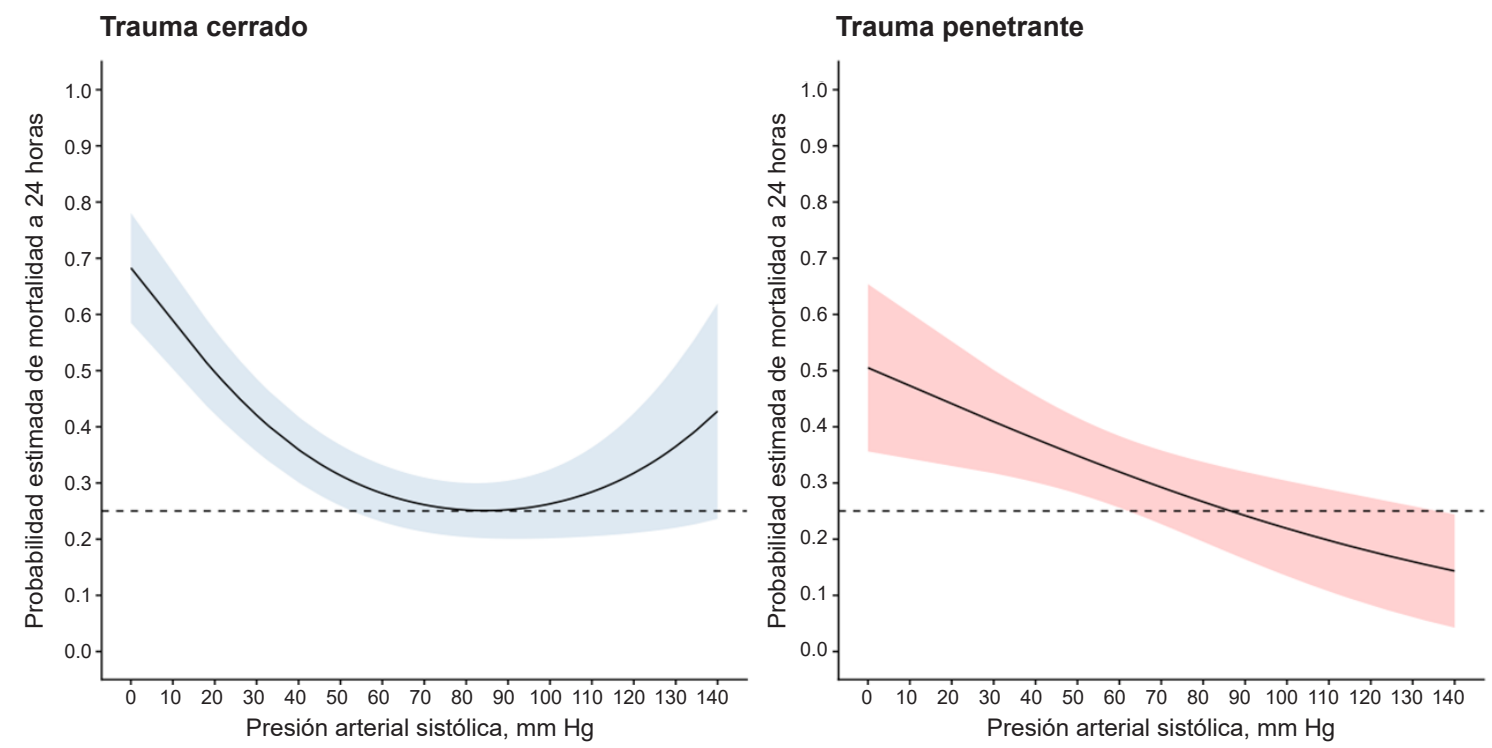

Línea negra corresponde a la mortalidad a 24 horas estimada y las áreas sombreadas representan el intervalo de confianza al $95 \%$. La línea punteada representa el punto de mortalidad del $25 \%$. La distribución de los datos de pacientes con trauma cerrado tiene una relación cuadrática y una prueba de Bondad de Ajuste de $p=0,531$, mientras en los pacientes con trauma penetrante tiene una relación lineal y una prueba de Bondad de Ajuste de $p=0,961$.

Figura 1. Mortalidad a 24 horas de acuerdo con la presión arterial sistólica previa a la oclusión aortica por REBOA. 

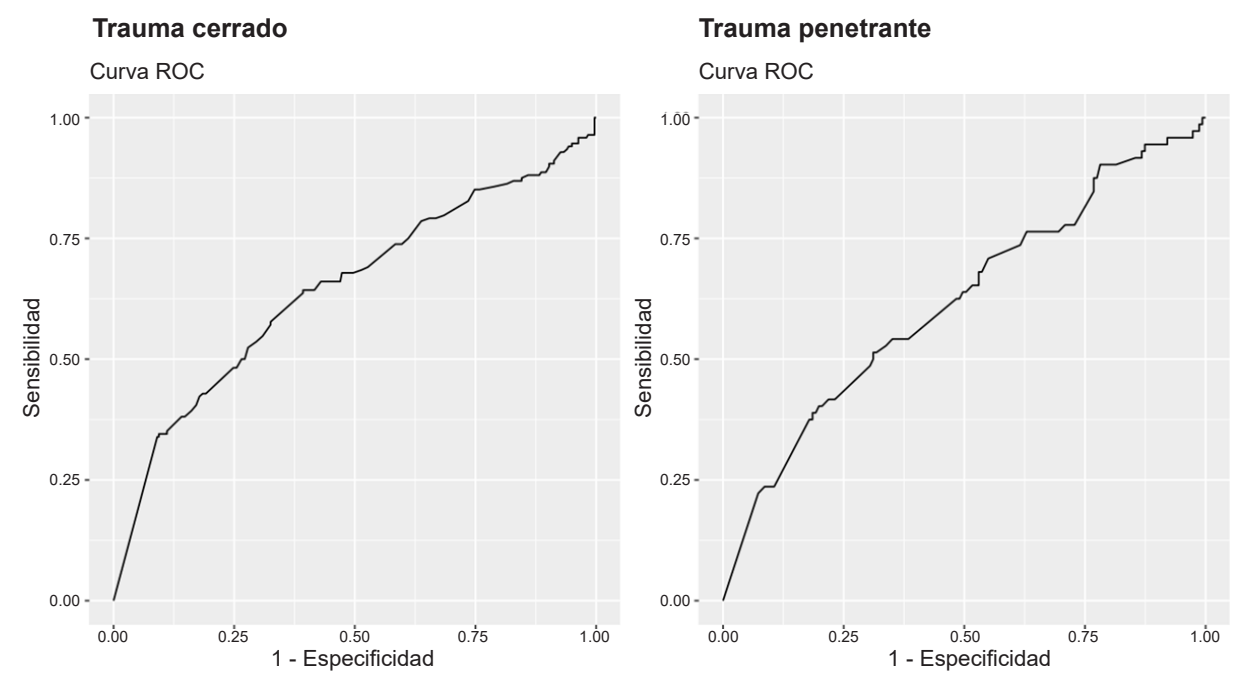

Figura 2. Curvas ROC de la presión arterial sistólica previa a la oclusión de la aorta con REBOA para la mortalidad a las 24 horas.

Tabla 4. Resumen de los puntos de corte de la presión arterial sistólica (PAS) previa al REBOA para predecir mortalidad en 24 horas

\begin{tabular}{lcccccccc}
\hline $\begin{array}{l}\text { Mecanismo } \\
\text { de Trauma }\end{array}$ & $\begin{array}{c}\text { PAS previo } \\
\text { al REBOA }\end{array}$ & Sensibilidad Especificidad & VPP & VPN & LR+ & LR- & OR (IC $\left.\boldsymbol{9 5}_{9 \%}\right)$ \\
\hline & $<60 \mathrm{~mm} \mathrm{Hg}$ & $64(56-71)$ & $61(55-66)$ & $48(41-55)$ & $75(69-80)$ & $1,62(1,35-1,95)$ & $0,60(0,48-0,74)$ & $2,71(1,80-4,09)$ \\
Trauma & $<70 \mathrm{~mm} \mathrm{Hg}$ & $74(66-80)$ & $42(36-47)$ & $42(36-47)$ & $74(66-80)$ & $1,26(1,11-1,44)$ & $0,63(0,47-0,84)$ & $2,00(1,30-3,11)$ \\
Cerrado & $<80 \mathrm{~mm} \mathrm{Hg}$ & $83(76-88)$ & $27(22-32)$ & $39(34-44)$ & $73(64-81)$ & $1,13(1,02-1,24)$ & $0,65(0,44-0,95)$ & $1,72(1,05-2,88)$ \\
& $<90 \mathrm{~mm} \mathrm{Hg}$ & $88(82-93)$ & $14(10-19)$ & $37(32-42)$ & $68(55-79)$ & $1,03(0,95-1,10)$ & $0,84(0,51-1,39)$ & $1,21(0,66-2,26)$ \\
& $<60 \mathrm{~mm} \mathrm{Hg}$ & $62(50-74)$ & $52(43-60)$ & $38(29-48)$ & $74(65-82)$ & $1,29(1,01-1,65)$ & $0,73(0,52-1,02)$ & $1,78(0,96-3,30)$ \\
Trauma & $<70 \mathrm{~mm} \mathrm{Hg}$ & $74(62-83)$ & $38(31-47)$ & $36(29-45)$ & $75(64-84)$ & $1,20(0,99-1,44)$ & $0,69(0,44-1,06)$ & $1,73(0,90-3,42)$ \\
Penetrante & $<80 \mathrm{~mm} \mathrm{Hg}$ & $85(74-92)$ & $23(17-31)$ & $34(27-42)$ & $76(61-87)$ & $1,10(0,97-1,26)$ & $0,66(0,36-1,22)$ & $1,67(0,76-3,90)$ \\
& $<90 \mathrm{~mm} \mathrm{Hg}$ & $92(83-97)$ & $15(9-21)$ & $34(27-41)$ & $79(59-92)$ & $1,07(0,97-1,18)$ & $0,57(0,24-1,35)$ & $1,87(0,69-5,91)$ \\
\hline
\end{tabular}

PAS: Presión arterial sistólica; VPP: Valor predictivo positivo; VPN: Valor predictivo negativo; LR: Likelihood ratio; OR: Odds ratio; IC: Intervalo de confianza

la aorta es aún incierto con la evidencia científica actual. La PAS ha sido usada como un parámetro clásico para determinar la necesidad de oclusión de la aorta ya que es una variable rápida y simple de medir. Sin embargo, la estimación de un punto critico de PAS previo a la oclusión de la aorta que indique al clínico sobre las posibilidades de mortalidad de los pacientes, es un tema en investigación actualmente ${ }^{9,10,14}$.

El presente estudio recopila la información de varios centros hospitalarios de Norte América, Suramérica, Europa, Asia y África, que han incursionado en el uso de REBOA para el manejo de pacientes con trauma severo. Este es uno de los estudios con el mayor tamaño de muestra, lo que permitió determinar que los puntos con mejor rendimiento estadístico para predecir mortalidad a 24 horas fueron en trauma cerrado una PAS de $41 \mathrm{mmHg}$ y para trauma penetrante una PAS de $47 \mathrm{mmHg}$.

No obstante, esos valores carecen de aplicabilidad en la clínica porque son valores con un alto riesgo de mortalidad y de paro cardiaco ${ }^{9}$. Por lo cual, se exploró la asociación univariada entre la mortalidad a las 24 horas y la PAS previa al REBOA, encontrando una relación cuadrática de estas variables en el trauma cerrado y una relación lineal en el trauma penetrante. Estos hallazgos son consistentes con estimaciones del riesgo de la PAS al 
ingreso en pacientes críticamente enfermos o con trauma severo ${ }^{18-20}$. Es de resaltar que la edad, la severidad del trauma y la localización anatómica de la lesión fue similar en los pacientes que fallecieron en las primeras 24 horas comparados con los supervivientes.

En el ambiente clínico, la definición de un valor óptimo de PAS debe cumplir con ser un punto por debajo del cual se asocia con un aumento del riesgo de la mortalidad. Es por ello que se propone a $70 \mathrm{mmHg}$ como el punto crítico de PAS previa al REBOA, dado que este valor se asocia con un aumento paulatino de la mortalidad estimada. Adicionalmente, esta cifra se asocia con una alta sensibilidad, pero regular especificidad en la predicción de mortalidad a las 24 horas, con un aumento en el numero de falsos positivos que potencialmente podría detectar, beneficiándolos con el uso de REBOA. Aunque la PAS tiene una moderada capacidad para predecir la mortalidad, esta debería complementarse con otros factores, como la respuesta o no a las maniobras de resucitación ${ }^{6,21}$.

Previamente, con datos de un único centro se estimaron los valores de PAS al ingreso asociados con un aumento de la mortalidad a las 24 horas y de paro cardiaco en pacientes sometidos a oclusión de la aorta, sin importar si fue por vía endovascular o abierta. Ellos reportaron que la PAS al ingreso de $60 \mathrm{mmHg}$ se asocia con un $50 \%$ de mayor probabilidades de mortalidad y la PAS al ingreso de $70 \mathrm{mmHg}$, con un probabilidad mayor de paro cardiaco ${ }^{14}$. Los datos de los registros REBOA muestran una mortalidad más conservadora, teniendo un punto de ascenso de la mortalidad estimada por encima del $25 \%$.

Por otro lado, existen factores que potencialmente podrían interactuar en los desenlaces clínicos como son la respuesta a los esfuerzos de la reanimación hemostática, tiempo transcurrido hasta lograr la oclusión de la aorta o del control definitivo de la lesión, requerimientos de transfusión de hemoderivados y comorbilidades, entre otros $^{22,23}$. La restauración oportuna del volumen sanguíneo en la atención inicial del trauma mejora la supervivencia de los pacientes y es una variable que aun está en investigación respecto a su papel en la resucitación del paciente manejado con REBOA ${ }^{24,25}$.

Dentro de las fortalezas de este estudio se encuentra que es la recopilación más grande de pacientes con trauma severo que fueron intervenidos con REBOA, de naturaleza multicéntrica, que permite tener un tamaño muestral que puede asociarse con mayor poder estadístico. Sin embargo, tiene limitaciones respecto a posibles diferencias en las definiciones de las variables o la falta de estandarización de atención del trauma entre los centros. Hay falta de uniformidad de la cuantificación de las variables de resucitación, como son el volumen de cristaloides o de hemocomponentes antes de la colocación del REBOA y en las primeras 24 horas. Adicionalmente, a pesar de que se reporta la mortalidad a las 24 horas, no se conoce la causa de la muerte o la fuente de la hemorragia.

\section{Conclusión}

Un valor de PAS de $70 \mathrm{mmHg}$ en pacientes con trauma severo y hemorragia no compresible del torso puede ser el punto crítico para la oclusión endovascular de la aorta, para mejorar la supervivencia de los pacientes, sin importar el mecanismo de trauma. Sin embargo, la PAS debe complementarse con otros factores clínicos para tomar la decisión oportuna.

\section{Cumplimiento de normas éticas}

Consentimiento informado: Este estudio está excluido de obtener consentimiento informado por parte de los pacientes incluidos en las bases de datos. Los centros colaboradores de las bases de datos obtuvieron su aval por parte de los comités institucionales y de ética previo a la recolección de datos de los pacientes.

Conflicto de interés: los autores declaran no tener ningún conflicto de interés.

Financiación: Este trabajo fue autofinanciado.

\section{Contribuciones de los autores:}

- Concepción y diseño del estudio: Elmer Palacios, Christian Delgado, Carlos Munar, Yaset Caicedo, Michael Parra, Carlos Ordoñez.

- Adquisición, diligenciamiento y validación de datos: Elmer Palacios, Christian Delgado, Carlos Munar, Yaset 
Caicedo, Alexander Salcedo, José Julián Serna, Fernando Rodríguez, Alberto García, Carlos Serna, Carlos Ordoñez.

- Análisis e interpretación de datos: Yaset Caicedo, Alexander Salcedo, José Julián Serna, Fernando Rodríguez, Alberto García.

- Redacción del manuscrito: Elmer Palacios, Yaset Caicedo, Christian Delgado, Michael Parra, Carlos Ordoñez.

- Revisión crítica: Alexander Salcedo, José Julián Serna, Fernando Rodríguez, Alberto García, Carlos Serna, Michael Parra, Carlos Ordoñez.

\section{Bibliografía}

1. Morrison JJ, Rasmussen TE. Noncompressible torso hemorrhage: a review with contemporary definitions and management strategies. Surg Clin North Am. 2012;92:843-58. https://doi.org/10.1016/j.suc. 2012.05.002

2. Kauvar DS, Lefering R, Wade CE. Impact of hemorrhage on trauma outcome: An overview of epidemiology, clinical presentations, and therapeutic considerations. J Trauma. 2006;60:S3-11. https://doi.org/10.1097/01.ta.0000199961.02677.19

3. Daban JL, Falzone E, Boutonnet M, Peigne V, Lenoir B. Wounded in action: the platinum ten minutes and the golden hour. Soins. 2014;788:14-5.

4. Meizoso JP, Ray JJ, Karcutskie CA, Allen CJ, Zakrison TL, Pust GD, et al. Effect of time to operation on mortality for hypotensive patients with gunshot wounds to the torso: The golden 10 minutes. J Trauma Acute Care Surg. 2016;81:685-91. https://doi.org/10.1097/TA.0000000000001198

5. Kuckelman JP, Barron M, Moe D, Derickson M, Phillips C, Kononchik J, et al. Extending the golden hour for Zone 1 resuscitative endovascular balloon occlusion of the aorta: Improved survival and reperfusion injury with intermittent versus continuous resuscitative endovascular balloon occlusion of the aorta of the aorta in a porcin severe truncal hemorrhage model. J Trauma Acute Care Surg. 2018;85:318-26. https://doi.org/10.1097/TA.0000000000001964

6. Ordoñez CA, Rodríguez F, Parra M, Herrera JP, GuzmánRodríguez M, Orlas C, et al. Resuscitative endovascular balloon of the aorta is feasible in penetrating chest trauma with major hemorrhage: Proposal of a new institutional deployment algorithm. J Trauma Acute Care Surg. 2020;89:311-9.

https://doi.org/10.1097/ta.0000000000002773

7. Manzano-Nunez R, Naranjo MP, Foianini E, Ferrada P, Rincon E, García-Perdomo HA, et al. A meta-analysis of resuscitative endovascular balloon occlusion of the aorta (REBOA) or open aortic cross-clamping by resuscitative thoracotomy in non-compressible torso hemorrhage patients. World J Emerg Surg. 2017;12:30. https://doi.org/10.1186/s13017-017-0142-5
8. Biffl WL, Fox CJ, Moore EE. The role of REBOA in the control of exsanguinating torso hemorrhage. J Trauma Acute Care Surg. 2015;78:1054-8. https://doi.org/10.1097/TA.0000000000000609

9. McGreevy DT, Abu-Zidan FM, Sadeghi M, Pirouzram A, Toivola A, Skoog P, et al. Feasibility and clinical outcome of reboa in patients with impending traumatic cardiac arrest. Shock. 2020;54:218-23. https://doi.org/10.1097/SHK.0000000000001500

10. Duchesne J, McGreevy D, Nilsson K, DuBose J, Rasmussen TE, Brenner M, et al. Delta Systolic Blood Pressure (SBP) can be a stronger predictor of mortality than pre-aortic occlusion SBP in non-compressible torso hemorrhage; an abotrauma and AORTA analysis. Shock. 2020. Volume Publish Ahead of Print. https://doi.org/10.1097/SHK.0000000000001560

11. Parks JK, Elliott AC, Gentilello LM, Shafi S. Systemic hypotension is a late marker of shock after trauma: a validation study of Advanced Trauma Life Support principles in a large national sample. Am J Surg. 2006;192:727-31. https://doi.org/10.1016/j.amjsurg.2006.08.034

12. Kristensen AKB, Holler JG, Mikkelsen S, Hallas J, Lassen A. Systolic blood pressure and short-term mortality in the emergency department and prehospital setting: A hospital-based cohort study. Crit Care. 2015;19:1-8. https://doi.org/10.1186/s13054-015-0884-y

13. Belenkiy SM, Batchinsky AI, Rasmussen TE, Cancio LC. Resuscitative endovascular balloon occlusion of the aorta for hemorrhage control: Past, present, and future. J Trauma Acute Care Surg. 2015;79:S236-42. https://doi.org/10.1097/TA.0000000000000770

14. Ordoñez CA, Rodriguez F, Orlas CP, Parra M, Caicedo Y, Guzmán-Rodríguez M. The critical threshold value of systolic blood pressure for aortic occlusion in trauma patients in profound hemorrhagic shock. J Trauma Acute Care Surg. 2020;89:1107-13. https://doi.org/10.1097/TA.0000000000002935

15. Meléndez JJ, Ordóñez CA, Parra MW, Orlas CP, ManzanoNúñez R, García AF, et al. Balón de reanimación endovascular de aorta para pacientes en riesgo de o en choque hemorrágico: experiencia en un centro de trauma de Latinoamérica. Rev Colomb Cir. 2019;34:124-31. https://doi.org/10.30944/20117582.106

16. Fluss R, Faraggi D, Reiser B. Estimation of the Youden Index and its associated cutoff point. Biometrical J. 2005;47:458-72.

https://doi.org/10.1002/bimj. 200410135

17. European Environment Information and Observation Network (Eionet). R Core Team. R: A language and environment for statistical computing. R Foundation for Statistical Computing. Vienna, Austria; 2020. Disponible en: http://www.r-project.org/index.html.

18. Bruns B, Gentilello L, Elliott A, Shafi S. Prehospital hypotension redefined. J Trauma. 2008;65:1217-21. https://doi.org/10.1097/TA.0b013e318184ee63 
19. Hasler RM, Nüesch E, Jüni P, Bouamra O, Exadaktylos AK, Lecky F. Systolic blood pressure below $110 \mathrm{mmHg}$ is associated with increased mortality in penetrating major trauma patients: Multicentre cohort study. Resuscitation 2012;83:476-81.

https://doi.org/10.1016/j.resuscitation.2011.10.018

20. Hasler RM, Nuesch E, Jüni P, Bouamra O, Exadaktylos AK, Lecky F. Systolic blood pressure below $110 \mathrm{mmHg}$ is associated with increased mortality in blunt major trauma patients: Multicentre cohort study. Resuscitation. 2011;82:1202-7. https://doi.org/10.1016/j.resuscitation.2011.04.021

21. Ordoñez CA, Khan M, Cotton B, Perreira B, Brenner M, Ferrada $\mathrm{P}$, et al. The Colombian experience in Resuscitative Endovascular Balloon Occlusion of the Aorta (REBOA): The progression from a large caliber to a low-profile device at a level I trauma center. Shock 2020. Publish ahead of print. https://doi.org/10.1097/SHK.0000000000001515

22. Brenner M, Teeter W, Hoehn M, Pasley J, Hu P, Yang $\mathrm{S}$, et al. Use of Resuscitative Endovascular Balloon
Occlusion of the Aorta for proximal aortic control in patients with severe hemorrhage and arrest. JAMA Surg. 2018;153:130-5.

https://doi.org/10.1001/jamasurg.2017.3549

23. Morrison JJ, Galgon RE, Jansen JO, Cannon JW, Rasmussen TE, Eliason JL. A systematic review of the use of resuscitative endovascular balloon occlusion of the aorta in the management of hemorrhagic shock. J Trauma Acute Care Surg. 2016;80:324-34. https://doi.org/10.1097/TA.0000000000000913

24. Duchesne JC, Kimonis K, Marr AB, Rennie K V, Wahl G, Wells JE, et al. Damage control resuscitation in combination with damage control laparotomy: A survival advantage. J Trauma. 2010;69:46-52. https://doi.org/10.1097/TA.0b013e3181df91fa

25. Duchesne JC, McSwain NE, Cotton BA, Hunt JP, Dellavolpe J, Lafaro K, et al. Damage control resuscitation: The new face of damage control. J Trauma. 2010;69:976-90. https://doi.org/10.1097/TA.0b013e3181f2abc9 